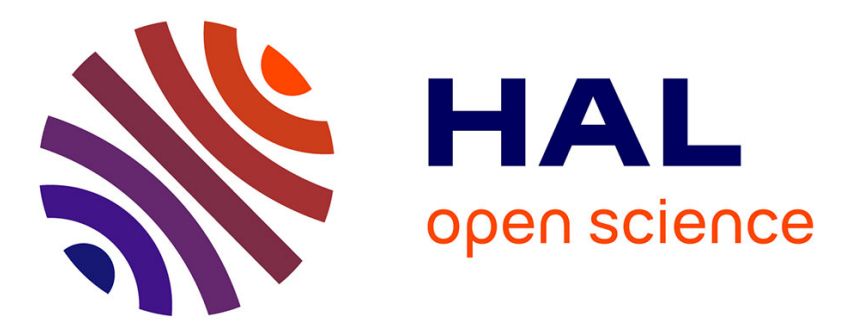

\title{
Comment communiquer lorsqu'on est un utilisateur infirme moteur cérébral (IMC) de type athétosique? Étude de cas des différents moyens de communication
} Yohan Guerrier, Christophe Kolski, Franck Poirier

\section{To cite this version:}

Yohan Guerrier, Christophe Kolski, Franck Poirier. Comment communiquer lorsqu'on est un utilisateur infirme moteur cérébral (IMC) de type athétosique? Étude de cas des différents moyens de communication. Ergo'IHM 2012, Oct 2012, Biarritz, France. pp.51-54. hal-00816556

\author{
HAL Id: hal-00816556 \\ https://hal.science/hal-00816556
}

Submitted on 22 Apr 2013

HAL is a multi-disciplinary open access archive for the deposit and dissemination of scientific research documents, whether they are published or not. The documents may come from teaching and research institutions in France or abroad, or from public or private research centers.
L'archive ouverte pluridisciplinaire HAL, est destinée au dépôt et à la diffusion de documents scientifiques de niveau recherche, publiés ou non, émanant des établissements d'enseignement et de recherche français ou étrangers, des laboratoires publics ou privés. 


\section{Comment communiquer lorsqu'on est un utilisateur infirme moteur cérébral (IMC) de type athétosique ? Étude de cas des différents moyens de communication}

\author{
Yohan Guerrier, Christophe Kolski \\ Univ Lille Nord de France, F-59000 Lille \\ UVHC, LAMIH, F-59313 Valenciennes \\ CNRS, UMR 8201, F-59313 Valenciennes \\ Yohan.guerrier@gmail.com \\ christophe.kolski@univ-valenciennes.fr
}

\author{
Franck Poirier \\ Laboratoire UMR Lab-STICC \\ Université de Bretagne Sud \\ F-56000 Vannes \\ Franck.Poirier@univ-ubs.fr
}

\begin{abstract}
RESUME
Cet article consiste en une étude à propos des différents moyens d'aide à la communication pour les utilisateurs de profil Infirme Moteur Cérébral (IMC) athétosique. Ils sont de trois types principaux : claviers virtuels, reconnaissance vocales et aides techniques physiques. La discussion permet de faire le lien sur les perspectives liées à un système d'aide à la communication destiné des utilisateurs de profil IMC athétosique.
\end{abstract}

\section{ABSTRACT}

This paper proposes a study of the various means of communication for users with cerebral palsy (CP) athetoid. Three main types of communication means are studied: virtual keyboards, voice recognition and technical physical helps. The discussion allows establishing a link with the perspectives: they concern the design of a communication support dedicated to users with $\mathrm{CP}$ athetoid profile.

\section{Author Keywords}

Communication; mobility; cerebral palsy athetoid BMI; slurred speech; communication aid

\section{ACM Classification Keywords}

H5.m. Information interfaces and presentation (e.g., HCI): Miscellaneous.

\section{General Terms}

Design, Languages

\section{INTRODUCTION}

La communication fait partie des actes importants effectués quotidiennement par tout être humain. La communication permet de développer des relations et de former sa propre personnalité, tout en accédant à l'information et la connaissance. Cependant, sur ce plan, tout le monde n'est pas égal, dans la mesure où nous pouvons réperto-

Permission to make digital or hard copies of all or part of this work for personal or classroom use is granted without fee provided that copies are not made or distributed for profit or commercial advantage and that copies bear this notice and the full citation on the first page. To copy otherwise, or republish, to post on servers or to redistribute to lists, requires prior specific permission and/or a fee.

Ergo'IHM 2012, October 16-19, 2012, Biarritz, France.

Copyright 2012 ACM 978-1-4503-1015-4/12/05...\$10.00. rier de nombreux handicaps, et ceci dans deux catégories distinctes : mental ; physique [12]. Dans cet article nous nous focalisons sur le handicap physique et plus spécialement sur les utilisateurs de profil IMC athétosiques (profil du premier auteur). Ces personnes ont pour particularité d'effectuer des mouvements involontaires, qui diminuent fortement la précision de leurs mouvements [11]. À l'intérieur des deux catégories, nous pouvons trouver des sous-catégories qui provoquent des troubles d'élocution. Concernant les handicapés mentaux, leurs problèmes résident dans le fait qu'ils éprouvent des difficultés pour formuler une phrase correcte. Par contre, à l'intérieur de la catégorie des handicaps physiques, nous pouvons trouver des sous-catégories qui engendrent des difficultés de prononciation. Les IMC athétosiques font partie des handicaps qui provoquent des troubles d'élocution : ces personnes n'ont aucun problème pour formuler des phrases; cependant les mots sortant dans leur bouche sont déformés à cause de la dysarthrie [15]. La loi $\mathrm{n}^{\circ}$ 2005-102 du 11 février 2005 [9] a pour objectif l'accessibilité pour les personnes handicapées aux établissements recevant du public et aux transports publics. Dans ce nouveau cadre légal, il faut trouver des solutions au problème de communication des personnes handicapées moteurs ayant des problèmes de prononciation. Dans ce contexte, il est important de proposer une aide à la communication pour les personnes IMC athétosiques. Dans la suite de cet article, nous allons présenter une étude de cas sur les différents types d'aide à la communication disponibles, suite à un retour d'expérience d'utilisation par le premier auteur. Puis nous effectuerons une synthèse associée à une discussion. Finalement, une conclusion et des perspectives termineront l'article.

ETUDE DES DIFFERENTS TYPES D'AIDE A LA COMMUNICATION RELATIVEMENT A DES UTILISATEURS IMC ATHETOSIQUE

Dans un premier temps, nous allons décrire différents claviers virtuels, puis nous présenterons certains systèmes de reconnaissance vocale et terminerons par les 
systèmes de saisie sans clavier virtuel. ${ }^{1}$ Les différents systèmes seront analysés comme suit: description du système, points forts puis points faibles, selon un retour d'expérience du premier auteur. Cette étude se base en effet sur les avis personnels et expériences de celui-ci (qui est représentatif d'utilisateurs de profil IHM athétosique, mais sans possibilité d'exhaustivité à ce sujet), qui a pu utiliser et tester lui-même, en tant qu'informaticien de formation, la plupart des systèmes recensés. Cet article est en quelque sorte un témoignage appelant d'autres études plus approfondies ou expérimentations.

\section{Claviers virtuels}

Les claviers virtuels permettent de remplacer les claviers physiques qui ne sont pas du tout adaptés pour les utilisateurs IMC athétosiques (ne pouvant pas utiliser de claviers physiques à cause de mouvements involontaires). On trouvera en Figure 1 un ensemble représentatif de claviers virtuels adaptés pour les personnes athétosiques, accessibles par joystick ou par défilement.
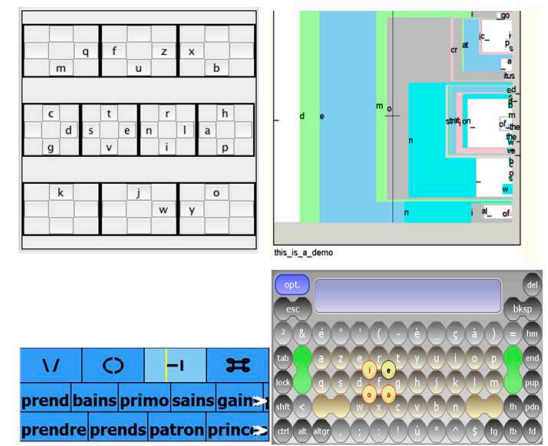

Figure 1. Quatre exemples de claviers virtuels : K-Thot optimisé (en haut, gauche), DASHER (haut, droite), HandiGlyph (bas, gauche), KEYGLASS (bas, droite)

K-Thot optimisé [1] (Fig. 1) correspond à un clavier virtuel composé de 4 touches ambigües. Pour saisir une lettre, l'utilisateur clique sur le bouton voulu et fait sortir la souris du côté de la lettre. Les lettres ont été regroupées par rapport à la fréquence d'apparition dans la langue française. Ce clavier permet de diminuer la distance parcourue par la souris. Le clavier demande un effort de concentration important pour trouver la lettre voulue, cela entraine une fatigue visuelle et mentale.

DASHER (présenté dans [10]) (Fig. 1) permet d'écrire des mots sans utiliser de clic. Au début de la saisie, le pointeur de la souris est au centre de l'écran, tandis que des lettres rangées dans des carrés de couleur se trouvent alignées tout à droite de la fenêtre ; écarter la souris vers la droite de la croix centrale lance le défilement des lettres vers la gauche de la croix. Selon le concepteur, on peut atteindre 30 mots à la minute, la prédiction de mot est efficace. Le mot proposé par le clavier est proche du

\footnotetext{
${ }^{1}$ Les systèmes de suivi de regard de type [8] ne seront pas traités dans cet article, aussi bien par manque de place qu'en raison du fait que, suite à de premiers tests, le premier auteur a trouvé le principe inadapté à son handicap à cause du nombre de mouvements involontaires trop élevé au niveau de la tête.
}

pointeur, cela diminue le parcours de la souris. La prise en main est difficile, demande une bonne vue et de la concentration pour trouver la lettre voulue.

HandiGlyph [3] (Fig. 1) repose sur une analogie avec les caractères latins en majuscules et sur la décomposition des lettres en forme de base appelées «primitives ». Il a été conçue pour permettre de saisir du texte avec seulement un clic par caractère, sur un appareil mobile. Ce procédé permet de réduire le nombre de clics durant la saisie. Cependant, l'utilisateur doit connaître la règle qui associe une des trois primitives à chaque lettre.

KEYGLASS (présenté dans [10]) (Fig. 1) offre, une fois qu'une lettre est saisie, quatre boutons semi-transparents représentant les lettres les plus probables. Il permet une diminution du parcours de la souris grâce au bouton qui apparaît autour de la touche sélectionnée. Cependant, le premier auteur de cet article constate une diminution de la vitesse de saisie à cause de l'absence de stabilité des propositions de lettres sur les boutons semi-transparents.

Métropolis [17] a été créé à partir de l'algorithme glouton du même nom, qui regroupe les lettres selon leur proximité à l'intérieur des mots, par rapport aux caractéristiques du langage (par exemple en français la lettre « $\mathrm{u} » \mathrm{a}$ de fortes probabilités de suivre la lettre $\langle\mathrm{q} »)$. L'organisation des touches permet de diminuer les mouvements de la souris et par conséquent l'effort physique durant la saisie. Cependant, l'utilisateur doit apprendre la position des lettres.

Chewing Word [5] (Fig. 1) possède un principe qui diffère largement des précédents; seules quelques lettres sont affichées simultanément, et s'agencent en fonction de la position du curseur lors de la dernière entrée. La saisie se fait par clic normal ou par clic long. La prédiction de mots est intégrée et dynamique, elle s'adapte au vocabulaire de l'utilisateur et il n'est même pas nécessaire de pointer le mot pour le valider, il suffit de faire un clic long sur la lettre. Le clavier virtuel demande de la concentration pour trouver la lettre voulue. Cela est dû au changement d'emplacement des lettres.

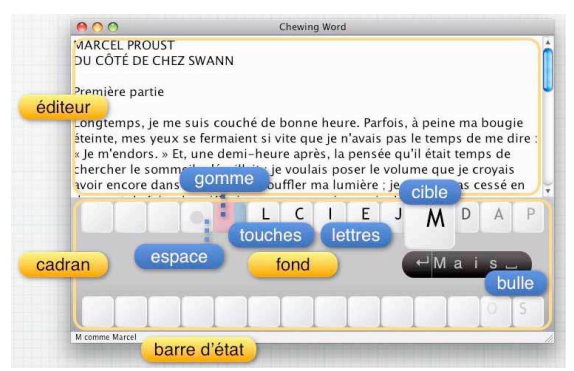

Figure 2. Chewing Word

Sibylle [14] est un clavier prédictif à défilement qui permet d'aider l'utilisateur à saisir plus rapidement, en prédisant les lettres et les mots les plus probables pendant la saisie. Grâce à la prédiction, l'utilisateur doit seulement saisir environ la moitié des lettres, par conséquent cela diminue de moitié le nombre de mouvements 
durant la saisie. Il faut un effort de concentration supplémentaire pour lire les propositions. Une fatigue mentale apparaît après quelques heures d'utilisation [14].

UKO-II (présenté dans [10]) est un clavier conçu pour les IMC, reprenant le principe du T9. Il regroupe les caractères de saisie en quatre zones numérotées, et il possède un système de désambiguïsation. Ce clavier possède seulement quatre touches; par conséquent cela diminue fortement la distance parcourue par la souris. Le fait de taper plusieurs fois sur le même bouton a perturbé le premier auteur car cela lui procure un stress supplémentaire dû au temps minimum pour sélectionner une lettre.

K-HERMES [6] est un clavier multitap reprenant la disposition des lettres des téléphones portables. L'utilisateur doit cliquer $\mathrm{N}$ fois pour saisir un caractère, $\mathrm{N}$ correspond au rang de la lettre sur le bouton. Ce clavier est destiné aux utilisateurs de type IMC. La réduction du nombre de boutons permet de diminuer le nombre de déplacements de la souris et de la fatigue physique. Le système multitap [10] ralentit la saisie à cause de l'augmentation du stress lié au temps limité pour saisir la lettre et à l'importance des erreurs de saisie, ces difficultés étant ressenties par le premier auteur.

\section{Les reconnaissances vocales pour les utilisateurs IMC}

Dans cette partie, nous allons décrire deux systèmes de reconnaissance vocale à l'attention des personnes handicapées moteurs de type IMC. Ces logiciels permettent de contrôler un ordinateur sans effort physique à propos des membres supérieurs, ce qui est a priori un atout majeur dans le domaine du handicap physique.

SPEECH BASED CURSOR CONTROL [4] découpe l'écran en zones. L'utilisateur les sélectionne en prononçant le numéro de la zone de façon récursive sur des zones de plus en plus petites jusqu'à atteindre la zone désirée. Ainsi l'utilisateur n'a plus à effectuer de mouvement avec les membres supérieurs. Ce phénomène se reproduit jusqu'à ce que l'utilisateur atteigne le bouton voulu. L'utilisation de la souris est facilitée pour les handicapés moteurs, car l'utilisateur le fait par la voix, par conséquent il n'effectue aucun mouvement avec ses membres supérieurs. Un inconvénient majeur est que le logiciel est difficilement utilisable par une personne ayant des difficultés d'élocution (cas fréquent pour les utilisateurs IMC athétosiques).

La reconnaissance vocale pour les IMC proposée par [13] est destinée aux personnes ayant une dysarthrie. Les chercheurs ont trouvé un algorithme permettant de transformer les ondes de la voix des personnes handicapées en ondes normalisées. La reconnaissance vocale devrait faciliter l'utilisation de l'outil informatique, car quasiment aucun mouvement physique n'est demandé. Cependant, ce système prometteur est encore en développement et test.
Systèmes de saisie physique pour les personnes handicapées physiques

Joystick EdgeWrite [16] est un système de saisie à l'aide des mouvements du joystick. L'utilisateur forme ses lettres en déplaçant un curseur à l'intérieur d'un carré de coin en coin avec le joystick. Ce procédé permet d'accélérer la saisie, car il n'y a pas de mouvement de souris. Cependant ce procédé convient aux utilisateurs IMC uniquement s'ils ne sont pas athétosiques.

Saisie avec deux boutons [2] : à l'écran, un clavier virtuel est représenté sous forme de rectangle; l'utilisateur dispose de deux boutons physiques, le premier pour les lignes et le deuxième pour les colonnes. Ce principe est destiné aux personnes lourdement handicapées moteur. Si l'utilisateur connait les coordonnées des lettres par cœur, la saisie peut être très rapide. La saisie demande un effort cognitif supplémentaire pour compter les coordonnées en ligne-colonne de chaque lettre.

Guide doigt [7] : ce système se compose d'une plaque en plastique perforée aux endroits des touches d'un clavier physique. Cela permet à l'utilisateur d'appuyer sur la touche voulue sans se tromper. Le principal avantage de ce système est le fait de pouvoir utiliser n'importe quel clavier physique pour lequel un guide doigt est disponible. Cependant, il ne concerne pas toutes les personnes athétosiques : cela dépend si la personne handicapée a appris à l'utiliser durant son enfance quand son cerveau possédait encore une plasticité importante [11].

\section{SYNTHESE ET DISCUSSION}

Nous avons décrit un ensemble de moyens, actuellement disponibles, d'aide à la communication. Chacun de ces systèmes possède un ensemble d'avantages et inconvénients, décrits précédemment, par rapport au type d'utilisateur visé dans l'article. Pour synthétiser, les claviers virtuels permettent de saisir de courts textes avec un niveau de fatigue relativement bas, alors que la reconnaissance vocale donne la possibilité aux personnes IMC athétosiques de saisir rapidement avec un effort physique moindre, mais sont pénalisés par leurs erreurs de reconnaissance plus ou moins fréquentes selon les systèmes et leurs contextes d'usage. Les autres systèmes recensés ont pour point fort le fait d'être indépendants vis-à-vis des matériels informatiques utilisés.

Les claviers virtuels ont pour inconvénient de ralentir la saisie et de demander un effort physique considérable lorsque les textes à saisir sont longs. La reconnaissance vocale est uniquement adaptée pour les personnes valides, mais des avancées récentes sont à suivre de près. Les autres systèmes demandent une précision trop importante dans les mouvements pour être utilisés quotidiennement par une personne IMC athétosique. Les auteurs de cet article pensent que les claviers virtuels resteront toujours un moyen lent et éprouvant pour les personnes athétosiques du fait des nombreux mouvements de la souris. La solution idéale serait une reconnaissance vocale complètement adaptée à leur manière de parler, 
mais les connaissances et technologies actuelles ne permettent pas de réaliser un système de communication vocale réellement opérationnel.

\section{CONCLUSION}

Dans cet article, nous avons étudié un ensemble de moyens de communication destinés à des utilisateurs de type IMC athétosique. Nous pensons que le clavier virtuel (disponible et commandable sur fauteuil roulant) est un outil bien adapté à la saisie, car sa taille peut être optimisée et demande peu de ressources. La reconnaissance vocale serait l'outil complémentaire idéal pour les utilisateurs IMC athétosiques, car elle permet d'effectuer des actions avec l'outil informatique sans faire d'effort physique à propos des membres supérieurs.

En conséquence, nos perspectives prennent la forme d'un système intégré d'aide à la communication entrant dans le cadre de la thèse du premier auteur; dénommé COMMOB (COMmunication et MOBility). Il aura pour but d'aider les personnes IMC athétosiques ayant des difficultés d'élocutions à communiquer (aussi bien avec des systèmes que des personnes), en situation de mobilité (dans les transports ou dans la rue) ou non. Pour ce faire, nous prévoyons d'utiliser des pictogrammes pour accélérer la saisie des phrases qui seront complétées si besoin à l'aide d'un clavier virtuel (pour les noms des villes, par exemple). Nous avons choisi les pictogrammes pour l'instant car les claviers virtuels ne permettent pas une saisie rapide et les reconnaissances vocales actuelles ne sont pas encore efficaces pour les personnes avec problèmes d'élocution. COMMOB sera sensible au contexte et utilisera des informations de géolocalisation; prenons l'exemple suivant : si l'utilisateur se trouve dans une gare alors COMMOB proposera prioritairement des pictogrammes en rapport avec une gare et des moyens de transports. De plus, la prédiction des pictogrammes sera affinée grâce à différentes informations récupérées sur internet (perturbations, grèves,...). Ce système est en cours de conception.

\section{REMERCIEMENTS}

Ce travail a été supporté par le ministère de l'Enseignement supérieur et de la recherche, le FEDER, CISIT, la Région Nord-Pas de Calais, l'ANR et le CNRS. Les auteurs remercient les relecteurs pour leurs remarques constructives. Le premier auteur remercie Ségolène Dumont qui a saisi sous sa dictée la première version de ce papier.

\section{BIBLIOGRAPHIE}

1. Baas, M., Guerrier, Y., Kolski, C., Poirier, F. Système de saisie de texte visant à réduire l'effort des utilisateurs à handicap moteur. Proceedings of the Ergonomie et Informatique Avancée Conference (12-15 october), ACM Press (2010), Biarritz, 19-26.

2. Baljko, B., Tam, A. Indirect Text Entry Using One or Two Keys. Proc. ASSETS'06, Portland, Oregon, USA, October 22-25, ACM Press (2006).
3. Belatar, M., Poirier, F. HandiGlyph : Une méthode de saisie de texte sur dispositifs mobiles pour personnes handicapées. Proceedings of IHM 08, ACM Press (2008), 81-84, September, Metz, France.

4. Dai, L., Goldman, R., Sears, A., Lozier, J. SpeechBased Cursor Control: A Study of Grid-Based Solutions. Proc. Assets '04, Atlanta, GA, USA, October 18-20, ACM Press (2004).

5. Grange, A. L'interface du clavier virtuel Chewing Word. Actes d'IHM'10, 22e Conférence Francophone sur l'Interaction Homme-Machine, September, ACM Press (2010), 237-240.

6. Guerrier, Y., Baas, M., Kolski, C., Poirier, F. Étude comparative entre un clavier virtuel AZERTY et un clavier multitap pour des utilisateurs souffrant d'une Infirmité Motrice Cérébrale de type tétraplégique athétosique. Proc. ASSISTH'2011, Paris, 148-155, janvier, 2011.

7. http://guidedoigts.free.fr/

8. Jacob, R.J.K., What you look at is what you get: eye movement-based interaction techniques. CHI '90, ACM Press (1990), 11-18.

9. http://www.legifrance.gouv.fr/affichTexte.do?cidText e=JORFTEXT000000809647\&dateTexte

10. Martin, B., Pecci, I. État de l'art des claviers physiques et logiciels pour la saisie de texte. RIHM, 8 (2) (2008), 147-205.

11.http://www.med.univ-

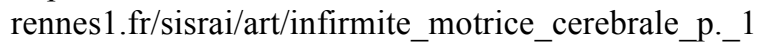
39-148.html

12.http://www.moteurline.apf.asso.fr/spip.php?rubrique3 9

13. Suanpirintr, S., Thubthong, N. The Effect of Pauses in Dysarthric Speech Recognition Study on Thai Cerebral Palsy Children. $i$-CREATe 2007, Singapore, April 23-26, 2007, 133-138.

14. Wandmacher, T., Béchet, N., Barhoumi, Z., Poirier, F., Antoine, J. Système Sibylle d'aide à la communication pour personnes handicapées : modèle linguistique et interface utilisateur. Proc. TALN 2007, Toulouse, 12-15 juin, 2007.

15.http://fr.wikipedia.org/wiki/Dysarthrie

16. Wobbrock, J.O., Myers, B.A., Aung, H.H., LoPrest H.F. Text Entry from Power Wheelchairs: EdgeWrite for Joysticks and Touchpads. ASSETS' 04, Atlanta, GA, USA, ACM Press (2004), 110-117.

17.Zhai, S., Hunter, M., Smith, B.A. The Metropolis Keyboard, An Exploration of Quantitative Techniques for Virtual Keyboard Design. UIST'2000, San Diego, CA, 119-12, ACM Press (2000). 